\title{
Serosurvey and Isolation of Infectious Bronchitis Virus in Chickens Reared in Commercial and Semi-Scavenging Systems
}

\author{
Himel Barua ${ }^{1}$, Paritosh Kumar Biswas ${ }^{*}$, M Nural Anwar ${ }^{2}$, Bhabesh Chandra Dey ${ }^{3}$ and Nitish Chandra Debnath ${ }^{1}$ \\ ${ }^{1}$ Department of Microbiology, Chittagong Veterinary University, Pahartali, Chittagong 4202, Bangladesh, ${ }^{2}$ Department of Microbiology, University of \\ Chittagong, Chittagong 4331, Bangladesh, ${ }^{3}$ Poultry Geneticist, Pahartali Zonal Poultry Farm (PZPF), Pahartali, Chittagong 4202, Bangladesh
}

[Received 15 May 2006; Accepted 07 October 2006]

\begin{abstract}
A cross sectional survey was undertaken to estimate the seroprevalence of infectious bronchitis virus (IBV) in chickens reared under both intensive and semi-scavenging systems in Bangladesh. Attempts were also made to isolate the virus from these two chicken populations. Chickens being reared under intensive system at a Government poultry farm (Pahartali Zonal Poultry Farm, PZPF, Chittagong, Bangladesh) and the semiscavenging system at Smallholder Livestock Development Project-2 (SLDP-2) areas, which is being operated in 5 southern districts of Bangladesh, were serologically investigated. The survey was conducted during the period from October 2005 to March 2006. Serum samples were at random from 184 chickens: 104 (Fayoumi and Rhode Island Red [RIR] breeds) from PZPF and 80 (Fayoumi, Sonali and indigenous) from the SLDP-2 areas. To isolate the IBV, organ samples were also collected from 100 dead chickens, 74 from PZPF and 26 from the SLDP-2 areas. Seroprevalence of the IBV in chickens was determined using Ab-ELISA test. Virological inocula prepared from the pooled organ samples of dead birds according to the standard procedure were inoculated through allantoic sac route (@ 0.2 ml inoculum/embryo) of 10-11 days' old developing-chicken embryos. The growth of the IBV was determined by finding characteristic changes in the embryos - curling and dwarfing at the age of $\mathbf{1 8}$ days. Allantoic fluids were harvested in positive cases. The survey revealed that, $19 \%$ (confidence intervals [CI] 11, 27\%) Fayoumi and 54\% (CI 35, 73\%) RIR chickens reared at PZPF were seropositive to the IBV. In semi-scavenging chickens, the seroprevalence was $98 \%$ (CI 96, $100 \%$ ), which is significantly higher $(p=\mathbf{0 . 0 0})$ than the chickens reared in the intensive system. Presence of the IBV was diagnosed in 11 and 6 dead chickens collected from PZPF and SLDP-2 areas, respectively.
\end{abstract}

Keywords: Infectious bronchitis virus (IBV), Serosurvey, Chicken

\section{Introduction}

Infectious bronchitis (IB), also referred to as avian infectious bronchitis caused by infectious bronchitis virus (IBV), is an acute highly contagious respiratory disease of chickens characterized by tracheal rales, coughing, and sneezing ${ }^{1}$. The disease is widely distributed and may cause considerable economic losses due to reduced weight gain, decreased egg production and diminished interior egg quality linked to downgrading of eggs ${ }^{1}$. Infectious bronchitis virus can infect chickens of all ages. Although the disease causes low mortality but concomitant infections especially with Mycoplasma gallisepticum and Escherichia coli can complicate the disease process resulting high mortality ${ }^{2-4}$. The clinical signs vary from mild to severe depending on the strains of the virus involved in the disease.

More than 20 serotypes the IBV have been identified worldwide based on the antibody cross-reactivity between the strains ${ }^{5-6}$ and the serotypes are antigenically distinct; that means, vaccine prepared from one serotype does not fully neutralize the others. Most common serotypes are Massachusetts, Connecticut 46, Arkansas 99, Iowa 97 and Gray ${ }^{1}$. Infectious bronchitis can be controlled by vaccination, and both live and killed IBV vaccines are commercially available in Bangladesh. However, without knowing the serotypic identity of the IBV, these vaccines are being used in private commercial poultry farms.

Before going to select the proper vaccine, determination of the antigenic spectrum of a particular serotype(s) of the IBV circulating in a region or country is crucial to get the maximum benefit. Published scientific information describing the serotypes of the IBV affecting chickens in Bangladesh is absent and reports on seroprevalence measuring the intensity of the IBV infections in chickens reared in government farms and semi-scavenging systems are scanty ${ }^{7-9}$. The present paper reports the findings of a preliminary study on the seroprevalence of the IBV in a Government poultry farm and in semi-scavenging chickens in a rural area of Bangladesh and attempted isolation of virus from dead chickens.

\section{Materials and Methods}

Nature of the study

It was a cross-sectional survey to determine the seroprevalence of the IBV in chickens raised in intensive and semi-scavenging

${ }^{*}$ Corresponding author:

Dr. Paritosh Kumar Biswas, Professor \& Head, Department of Microbiology, Chittagong Veterinary University, Pahartali, Chittagong 4202, Bangladesh

Tel (Office): (031) 659093/110, 126; Fax: +880 (031) 659492; E-mail: biswaspk2000@yahoo.com 
systems in Bangladesh. Additionally, organ samples collected from deceased chicken of these two populations were analysed to isolate the IBV in embryonated chicken eggs. The study was conducted during the period from August 2005 to March 2006. All laboratory tests were undertaken at the Department of Microbiology, Chittagong Veterinary University (CVU), Chittagong, Bangladesh.

\section{Study population}

Chickens reared at Pahartali Zonal Poultry Farm (PZPF), Chittagong, and in semi-scavenging system in Chatkhil Upazila of Noakhali district (under the Smallholder Livestock Development Project-2 area, SLDP-2) were randomly investigated. In PZPF, Fayoumi (mother line) and Rhode Island Red (RIR) chickens are reared in intensive system to produce the crossbred chickens, 'Sonali' ( $F_{1}$ generation). The Sonali as well as the Fayoumi chickens were distributed among the smallholder under the DLSsupported (and international-donors assisted) different programmes. SLDP-2 was one of these programmes operating its activities in 26 Upazilas of five southern districts of Bangladesh. In the SLDP-2 areas, most of the smallholders (about 96\%) of poultry were called 'key-rearers' who rear Sonali, Fayoumi and Deshi (indigenous, non-descriptive) chickens. Altogether, there were 4,000 key-rearers in Chatkhil Upazila under the SLDP-2. Commonly, the key-rearers under the SLDP-2 area got their chickens vaccinated only against Newcastle disease. Unlike the SLDP-2 areas, the chickens in PZPF are vaccinated against at least four diseases, viz., Newcastle disease ND), infectious bursal disease (IBD), fowl pox, fowl cholera and Marek’s disease.

\section{Estimation of required sample size and collection of samples}

A flock of chickens (strength 5,000 birds) at PZPF and 25 keyrearers' small-flocks (median number of chickens $=9$ ) in the Chatkhil Upazila were selected at random. For serological investigation, the minimum sample size was 25 for the flock of PZPF and 4 for each key-rearers' households of Chatkhil Upazila based on the formula ${ }^{10}, \mathrm{n}=\left\{1-(1-\mathrm{C})^{1 / \mathrm{D} x \text { sens }}\right\}\{\mathrm{N}-0.5(\mathrm{D}-1)\}$, where $\mathrm{n}=$ the required number of samples, $\mathrm{C}=$ the desired certainty (here, 95\% i.e. 0.95), sens = estimation of test sensitivity (here, $55 \%$ i.e. 0.55 ), $\mathrm{D}=$ the prevalence considered (here, $25 \%$ i.e. 0.25 ) within the chicken population $\mathrm{x} N$ (here, $\mathrm{N}=5000$ in case of PZPF, and $\mathrm{N}=9$ in case Chatkhil Upazila). Using non-probabilistic sampling, organ samples were collected from dead chickens at random of the total number of deaths encountered in these two populations during the study.

\section{Sample collection and investigation}

Blood samples were collected aseptically from the brachial vein of the chickens. When separated from blood clots a serum sample was transferred to a sterile 2.5-ml Eppendorf tube and then preserved chemically with merthiolate at a concentration of 1:10,000 to prevent bacterial contamination. Each Eppendorf tube was marked with the name of the breed of the bird, its age and the date of collection of the serum sample. The serum samples were kept frozen at $-85^{\circ} \mathrm{C}$. Organ samples like trachea, lungs, kidneys, caecal tonsils and cloaca were collected from the dead chickens in separate polythene-bags and all samples taken from an individual bird were enclosed within another polythene bag that was tagged with the identification number of the bird. The samples were stored at $-85^{\circ} \mathrm{C}$ until investigated.

\section{Serological investigation}

Antibody-capture ELISA was used to estimate seroprevalence of the IBV in chickens reared at PZPF and SLDP-2 areas. The test kit was obtained from Bio-check, Holland. The test was performed according to the manufacturer's instruction provided with the test kit. At the end of the assay, the wells of the plates were read at $405 \mathrm{~nm}$ in an ELISA plate reader (Statfax Co, USA). To estimate antibody titres from the absorbance values, the following standard curve equation (given by the manufacturer) was used: $\log _{10}$ titre $=1.09\left(\log _{10} \mathrm{~S} / \mathrm{P}\right)+3.36$, where $\mathrm{S} / \mathrm{P}=$ ratio of the absorbance values of a test sample and the positive control. For the status of the IBV in chickens, serum samples with $S / P$ value $\geq 0.2$ (titre $\geq 834$ ) 0.150-0.199 (titre 625-833) and $\leq 0.149$ (titre $\leq 624$ ) were considered as seropositive, suspected and negative, respectively.

\section{Isolation of the IBV in embryonated chicken-eggs}

Virological inoculum was prepared using the pooled-homogenized organ samples (collected from each dead chicken) in antibioticPBS (phosphate-buffer saline). From each inoculum prepared 0.2 $\mathrm{ml}$ was inoculated into each of two 10-11-days-old chicken embryos through the route of allantoic sac following the technique of Senne ${ }^{11}$. Seven days after inoculation, the eggs were examined for two gross characteristic changes of embryos, curling and dwarfing, which are the characteristic gross changes produced by the IBV in chicken embryos ${ }^{1}$. The allantoic fluids from the eggs exhibiting characteristic changes were harvested and preserved at $-85^{\circ} \mathrm{C}$ for future studies.

\section{Data analysis}

The S/P value obtained for each test sample was entered in the spread-sheet programme (MS EXCEL) and the results were interpreted according to the ranges of values of $\mathrm{S} / \mathrm{P}$ of the test samples. Seroprevalence of the IBV was calculated as the number of positive cases divided by total number of birds tested. Significance of the difference of the IBV seropositive cases in the two study populations was calculated using a $\chi^{2}$ test.

\section{Results}

Table 1 shows the number of serum samples collected and the seroprevalence of the IBV in chickens reared in both intensive and semi-scavenging systems. Altogether, blood sera were collected and analysed from 184 chickens, of which 104 and 80 were sampled from chickens reared in the intensive and the semiscavenging system, respectively. The seroprevalence of the IBV was higher in the semi-scavenging chickens compared to the chickens reared in the intensive system in PZPF $(p<0.05)$. More than $90 \%$ of both Deshi (indigenous) and exotic chickens, reared in the semi-scavenging system, were seropositive to the IBV. However, the difference between these two groups was insignificant $(p>0.05)$. Conversely, the lowest seroprevalence of the IBV was recorded in the Fayoumi chickens and the magnitude of the seroprevalence of the IBV between the Fayoumi and RIR chickens reared at PZPF differed $(p<0.05)$. 
Table 1. Seroprevalence of infectious bronchitis virus (IBV) in chickens reared in the intensive and in the semi-scavenging systems

\begin{tabular}{llccc}
\hline Chicken & System of rearing & No. & No. (\%) of seropositive cases & 95\% CI \\
\hline Fayoumi & Intensive $^{\mathrm{I}}$ & 78 & $15(19)$ & 11,27 \\
RIR & Intensive $^{\mathrm{I}}$ & 26 & $14(54)$ & 35,73 \\
Fayoumi + Sonali & Semi-scavenging $^{\mathrm{S}}$ & 31 & $29(94)$ & 88,100 \\
Deshi & Semi-scavenging $^{\mathrm{S}}$ & 49 & $48(98)$ & 96,100 \\
Total & - & 184 & $106(58)$ & 50,66 \\
\hline
\end{tabular}

RIR = Rhode Island Red breed; Deshi = Indigenous, non-descriptive variety; $\mathrm{CI}=$ Confidence intervals; $\mathrm{I}=$ chickens reared at Pahartali Zonal Poultry Farm (PZPF), Chittagong; S = chickens reared in the Chatkhil Upazila, Noakhali

Organ samples were collected from 100 chickens from the PZPF and the Chatkhil Upazila during this study (Table 2). Seventeen samples elicited characteristic embryonic changes suggestive of the IBV.

Table 2. Presence of infectious bronchitis virus (IBV) in dead chickens investigated from PZPF and the Chatkhil Upazila

\begin{tabular}{lcc}
\hline Place of investigation & $\begin{array}{c}\text { No. chickens } \\
\text { investigated }\end{array}$ & $\begin{array}{c}\text { No. positive } \\
\text { for the IBV }\end{array}$ \\
\hline PZPF & 74 & 11 \\
Chatkhil Upazila & 26 & 6 \\
Total & 100 & 17 \\
\hline
\end{tabular}

PZPF = Pahartali Zonal Poultry Farm

Figure 1 portrays the characteristic embryonic changes supposed to be caused by the IBV. The two curled and dwarfed embryos, which were of 18 days of age, are shown in the picture straddling a normal one (of the same age) placed on the centre.

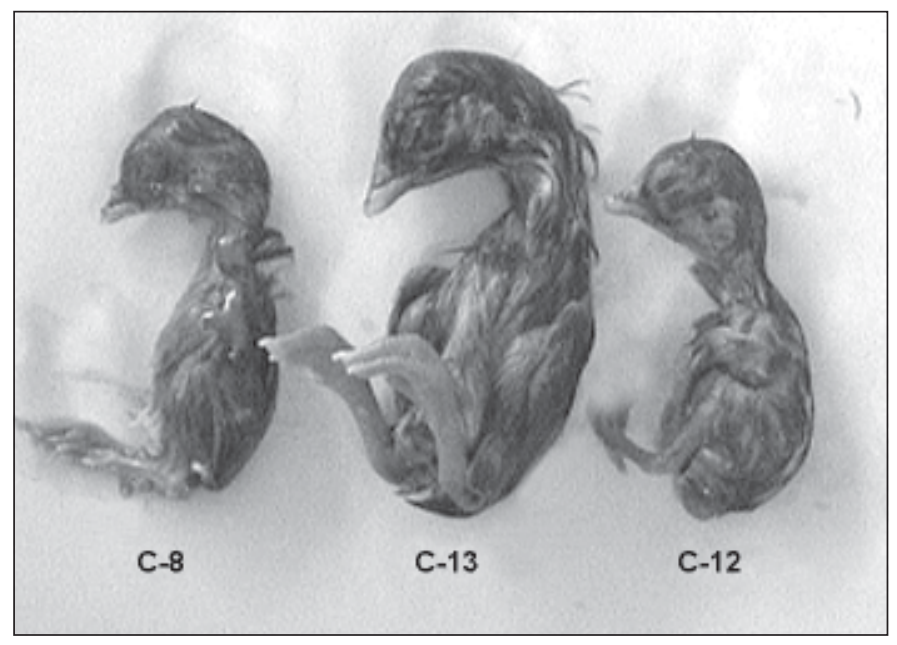

Figure 1. Gross changes in chicken embryos suspected to be caused by infectious bronchitis virus (IBV) at 18 days of age $(C-8, C-12=$ curled and dwarfed embryos; $C-13=$ a normal embryo).

\section{Discussion}

The results of this study indicate a high seroprevalence of the IBV in semi-scavenging chickens reared in the SLDP-2 areas. Even the low seroprevalence of the IBV in chickens raised under intensive system also suggests the endemic nature of the virus in that population. The high seroprevalence of the IBV in the semiscavenging chickens found in this study is an agreement with Alam et al. ${ }^{7}$ and Biswas ${ }^{9}$ who reported the seroprevalence to be $92.60 \%$ and $77.83 \%$, respectively.

Both Fayouni and Rhode Island Red (RIR) breed chickens were reared in the Pahartali Zonal Poultry Farm (PZPF) and the age of these chickens was the same. About $50 \%$ of RIR chickens were seropositive to the IBV; however, only 20\% Fayoumi were seropositive. Because they were reared in two different sheds. This difference could be a localized reflection due to isolation of these chickens in two separate. The seropositivity of chickens to the IBV does not mean that these birds were infected with the virus and consequently developing the disease. The birds that were seropositive to the IBV either had been infected or exposed to the IBV because they were not vaccinated against IB.

It was beyond the scope of the study to find out the holistic causes/factors involved in the deaths of chickens that had been investigated for the presence of the IBV. While acknowledging these limitations, we suspected that at least 17 of them were harbouring the IBV before the deaths. For them, the virus could have a contributory role to the deaths. In addition, the IBV can cause high mortality in chickens if the birds are concomitantly infected with the IBV and other pathogens particularly Mycoplasma gallisepticum (MG) and Escherichia coli ${ }^{2-4}$. We didn't investigate whether any of these 17 chickens were simultaneously affected with other pathogens. But there are reports on the high incidences of colibacillosis (21.48\%) and mycoplasmosis (61.74\%) in semi-scavenging chickens reared in the SLDP-2 areas $^{9}$ and the high seroprevalence (>90\%) of the IBV in these chickens creates the background of the speculation that some of them could have died as a synergistic action between or among the three pathogens-IBV, MG and E. coli.

Curling and dwarfing of the chicken embryos are considered as positive changes caused by the IBV, however, any further serological or virological confirmation was not attempted. Isolation of the IBV in embryos often requires up to 4 blind passages $^{1}$. Due to limitation of resources we recorded the embryonic changes at the end of first or at best second passages. The number of positive cases for the IBV in dead chickens could have been more instead of 17 if we went on giving passages the of the materials in embryonated eggs a few more times. However, 
based on the results of this study it is evident that the IBV is present in the study populations that encompassing chickens reared in both intensive and semi-scavenging systems. The results of the serological survey also disclose the high seroprevalence ( $>90 \%$ ) of the IBV particularly in chickens reared in the semiscavenging system. Therefore, vaccination against the IBV for these chickens may be considered. However, the proper vaccine for these chickens should be chosen only when the stereotypic identity of the circulating virus(s) is known. The allantoic fluids as crude sources of the field viruses had already been harvested and preserved. These preserved samples have to be studied further to isolate and characterize the virus.

\section{Acknowledgement}

This study was a part of an on going MPhil (in Microbiology) programme been undertaken at the Department of Microbiology, University of Chittagong and the Department of Microbiology, Chittagong Veterinary University, which was financed by the DANIDA-SLDP-2 Fellowships. We thank the financing agencies for supporting the study.

\section{References}

1. Cavanagh D \& Naqi S. 2003. Infectious bronchitis. In Diseases of Poultry (Saif YM, Barnes JH, Fadley AM, Gilson JR, McDougald LR \& Swayen DE eds), $11^{\text {th }}$ edn, 101-119. Iowa State University Press, Ames, Iowa.

2. Hokonohara S, Dekuras Y, Kanbayashi A, Tsutsui M, Sato T \& Sato S. 2004. Effect of combined eradication program for establishing mycoplasma eradication program for establishing mycoplasma (MG, MS) free breeding flocks of layer chickens. Abstract Book, p 742. Proceedings of the $22^{\text {nd }}$ World's Poultry Congress, Istambul, Turkey, $8^{\text {th }}-13^{\text {th }}$ June 2004. Istambul, Turkey.
3. Nakamura K, Cook JKA, Frazier JA \& Narita M. 1992. Escherichia coli multiplication and lesions in the respiratory tract of chickens inoculated with infectious bronchitis virus and/or Escherichia coli. Avian Dis. 36: 881-890.

4. Rodrigues OD, Nascimento E, Do R, Tortelly R, Vogel J, Nascimento M, Do F, Lignon GB \& Mendonca GADE. 2001. Damages caused on broiler chickens by the induced action of M. gallisepticum and E. coli. Vet Bulletin. 72(7): Abstract No. 5023.

5. Cowen BS \& Hitchner SB. 1975. Serotyping of avian infectious bronchitis viruses by the virus-neutralization test. Avian Dis. 19: 583-595.

6. Hopkins SR \& Yoder HW Jr. 1974. Increased incidence of airsacculitis in broilers infected with Mycoplasma synoviae and chicken-passaged infectious bronchitis vaccine virus. Avian Dis. 28: 386-396.

7. Alam J, Koike I, Giasuddin M \& Rahman MM. 2003. Seroprevalence of poultry diseases in native chickens in Bangladesh. Bangladesh Society for Veterinary Education \& Research (BSVER), Publication No. 24, p 26. Bangladesh Agricultural University, Mymensingh.

8. Bhattacharjee PS, Majumder, JU, Miah, AH, Ali MI \& Rahman MM. 1996. Prevalence of infectious bronchitis antibodies in chickens in Bangladesh. Bangladesh Vet J. 30(1-2): 1-06.

9. Biswas PK. 2004. A longitudinal study to identify the causes on the mortality of 'Sonali' birds and broody hen chicks of key beneficiaries in the SLDP-2 area. Final Report, p 78. Smallholder Livestock Development Project-2 (SLDP-2) Applied Research Project, Chittagong, Bangladesh.

10. Cannon RM \& Roe RT. 1982. Livestock Disease Surveys: A Field Manual for Veterinarians. Australian Government Publishing Service, Canberra.

11. Senne DA. 1989. Virus propagation in embryonating eggs. In A Laboratory Manual for the Isolation and Identification of Avian Pathogens (Purchage HG, Arp LH, Domermuth CH \& Pearson JE), $3^{\text {rd }}$ edn, pp 176-181. American Association of Avian Pathologists, Pennsylvania. 